\section{Bericht und Beschlüsse der ausserordentlichen Ärztekammer vom 2. Februar 2000}

\author{
F.-X. Deschenaux, Generalsekretär der FMH
}

Diese ausserordentliche Ärztekammersitzung diente ausschliesslich dazu, über die Anträge zur Tarifstruktur TarMed, den Arzttarif UV/MV/IV sowie das Entscheidfindungsverfahren betreffend der Einführung der neuen Tarifstruktur in den kantonalen Tarifen des KVG zu verhandeln und Beschluss zu fassen.

Die Sitzung wird, nachdem das notwendige Quorum erreicht ist, um 09.40 Uhr eröffnet. Der Präsident, Dr. H. H. Brunner, informiert die Versammlung, dass zwei Gäste bei den Verhandlungen zugegen sind, Dr. M. Ganz, Präsident der Schweizerischen Vereinigung der Belegärzte an Privatspitälern SVBP/ ASMI sowie Fürspr. F. Bernath, Generalsekretär der Foederatio Medicorum Scrutantium (FMS). Die Ärztekammer erteilt stillschweigend dem Präsidenten die Erlaubnis, dass diese beiden Gäste das Wort ergreifen können. Es wird weiter festgestellt, dass einige Anträge nach der festgelegten Frist eingegangen sind und deshalb in den Unterlagen an die Delegierten nicht mehr Eingang finden konnten; die Rücksprache mit den betroffenen Antragstellern hat jedoch ergeben, dass sich ihre Anträge entweder mit anderen, fristgerecht eingereichten Anträgen deckten oder sich problemlos in die Verhandlungen würden einbeziehen lassen. Der Präsident macht die Delegierten bereits an dieser Stelle darauf aufmerksam, dass die Abstimmung über die Tarifstruktur Alpha 2.2 spätestens um 15.30 Uhr stattfinden muss, unabhängig davon, wie weit die Verhandlungen zu diesem Zeitpunkt fortgeschritten sein werden.

Nachdem auch noch die sechs Stimmenzählerinnen und Stimmenzähler bezeichnet wurden, steigt man in die Verhandlungen ein.

\section{Bereinigung der statutarischen Voraussetzungen für eine allfällige Urabstimmung}

Der Präsident verweist auf die vorliegende schriftliche Information zuhanden der Delegierten betreffend der Zusammensetzung dieser ausserordentlichen Ärztekammer sowie einer allfälligen Urabstimmung und stellt, nachdem niemand das Wort verlangt, fest, dass diese schriftlichen Erläuterungen nicht bestritten werden und demzufolge als stillschweigend genehmigt erklärt werden können.

\section{2. Übersicht über den Stand der Verhandlungen und die politische Lage}

Der Präsident erinnert daran, dass die zweite Hälfte 1999 ausschliesslich dazu gedient hat, die Version Beta 3 in eine Version "Alpha" der Tarifstruktur umzusetzen (heute Version "Alpha 2.2»). In dieser Umsetzungsphase wurden von den Verhandlungspartnern auch einige Forderungen des Preisüberwachers berücksichtigt. Für diese Arbeiten mussten beträchtliche Ressourcen an Personal, Finanzen und Zeit mobilisiert werden, wobei vor allem Letzteres dafür verantwortlich war, dass auf die ursprünglich vorgesehene Durchführung einer Ärztekammersitzung im Dezember 1999 verzichtet werden musste. Die Verhandlungspartner haben dabei intensive Diskussionen geführt zu für den künftigen Tarif so kruzialen Fragen wie Dignität, «Handling» des Tarifs sowie Verträge über und im Zusammenhang mit der künftigen Tarifstruktur (UV/MV/IV-Arzttarif; Rahmenvertrag zwischen dem Konkordat Schweizerischer Krankenversicherer [KSK] und der FMH über die Einführung der neuen Tarifstruktur in den Kantonen). Auch die Kostenneutralität hat selbstverständlich die Verhandlungspartner beschäftigt, wobei die zentrale Frage hier der Taxpunktwert im Moment des Inkrafttretens der Tarifstruktur ist. Ein weiterer wichtiger Verhandlungspunkt: die Nachfolgeorganisation TarMed, da TarMed nach Erfüllung ihrer Aufgaben keine Daseinsberechtigung mehr hat.

Zur Frage der "Feldstudien" und der "Pilotstudien" liegt dem Präsidenten daran, einige ihm unbedingt notwendig scheinenden Präzisierungen vorzunehmen. Der Begriff "Feldstudie» ist ein relativ offener Begriff und wird dort angewendet, wo Leistungserbringer nach dem neuen Tarif Abrechnungen vornehmen, der alte Tarif aber der allein anwendbare bleibt. Solche Studien wurden bereits und werden noch durchgeführt und sie werden sicher einige nützliche Informationen liefern. Pilotstudien hingegen dienen dazu, den neuen Tarif in einem klar definierten zeitlichen Rahmen anzuwenden; solche Studien machen nur Sinn, wenn sämtliche Akteure (sämtliche Ärztinnen und Ärzte einer Stadt, Spitalärztinnen/ -ärzte, Ärztinnen und Ärzte eines Kantons) mitmachen. Es war ursprünglich vorgesehen, solche Pilotstudien in einigen Kantonen (8) durchzuführen; darauf musste aber verzichtet werden, weil der Wille nicht bei allen Betroffenen vorhanden war und/oder weil die notwendigen finanziellen Mittel fehlten. Die TarMed hat feststellen müssen, dass solche Pilotstudien nicht durchführbar sind und es deshalb keinen Sinn macht, weiterhin darauf zu bestehen.

Der Präsident fährt in seinem Einführungsreferat fort, indem er die Delegierten um Verständnis dafür bittet, dass der Zentralvorstand - auch wenn jetzt noch keine für eine definitive Entscheidung ausgereiften Papiere vorliegen - von ihnen Hinweise erwartet, welche Positionen die FMH verteidigen soll in bezug auf die mit der Tarifstruktur eng verbundenen Punkte wie Kostenneutralität, noch abzuschliessende 
Verträge und, falls notwendig, Verhalten gegenüber gewissen staatlichen Institutionen.

Der Präsident informiert weiter darüber, dass eine interne Evaluation über die Ergebnisse der letzten Verhandlungen zwischen den Partnern stattgefunden hat, welche für die finale Redaktion der Version "Alpha 2.2" eine wichtige Rolle spielte. Es ist ebenfalls eine externe Evaluation vorgesehen, deren Ergebnisse kurzfristig zu erwarten sind und die, was die Faktoren betrifft, welche integraler Bestandteil der technischen Leistung sind, insbesondere im Spitalbereich, bereits für die Überarbeitungsrunde 2000 zur Anwendung kommen können. Das Mandat sollte einem ausländischen Institut übertragen werden, welches auf ökonomische und tarifarische Fragen sowie Spitalmanagement spezialisiert ist.

Der Präsident gibt zum Abschluss seines Einführungsreferates in aller Klarheit zu verstehen, dass die von der Ärztekammer am heutigen Tag erwartete Entscheidung, keine Abstimmung über die Kostenneutralität, sondern eine Abstimmung über die Tarifstruktur ist. Der Beschluss muss klar sein, die Alternative kann nur "Ja" oder "Nein" sein, denn ein "Ja aber» wird als ein «Nein» verstanden werden. Er erinnert daran, dass gesetzliche Fristen seit langem überschritten und dass alle Verträge (UV/MV/IV-Arzttarif, Kantonale Vereinbarungen, Spitalleistungskatalog) gekündigt sind. Er wiederholt an dieser Stelle nochmals, dass die Abstimmung über die Tarifstruktur in keiner Art und Weise ausschliesst, dass dem Zentralvorstand und seiner Verhandlungsdelegation Hinweise betreffend der $\mathrm{zu}$ verteidigenden Positionen in den wichtigen und jetzt noch offenen Fragen (Kostenneutralität, Rahmenvertrag, usw.) geliefert werden.

\section{Ergänzende Konzepte und Vereinbarungen zu Tarifstruktur Version "Alpha 2.2"}

Bevor man in die Verhandlungen über die Kostenneutralität eintritt, muss sich die Ärztekammer mit einem Antrag (Nr. 1 zu Traktandum Nr. 3, 5 und 6) von Dr. R. Streit, dem Präsidenten der Ärztegesellschaft des Kantons Bern, befassen, dessen Ziff. 3, falls angenommen, einer generellen Rückweisung der unter den Ziffern 3.1 bis 3.7 von Traktandum 3 zur Beschlussfassung unterbreiteten Konzepte und Vereinbarungen gleichkäme und man demzufolge unmittelbar auf den nächsten Traktandenpunkt eintreten würde.

Dr. R. Streit erklärt, er vertrete die Meinung zahlreicher Mitglieder und plädiere für die Rückweisung sämtlicher Vorlagen, die noch nicht abschliessend ausgehandelt seien. Mehr als tausend Positionen seien für die Revisionsrunde 2000 vorgesehen, zwei Kapitel seien zu überarbeiten, die Entschädigungen für operativ-interventionelle Leistungen müssten nochmals überprüft werden. Wie man sich in einem solchen Wust von Unklarheiten binden könne, fragt sich der Redner und hält es für absolut unverantwortlich, eine Urabstimmung über die Tarifstruktur ins Auge zu fassen, solange weder die Gefahr des Damoklesschwertes der Kostenneutralität gebannt noch die margenunabhängige Medikamentenabgabe (MUMA) einer Lösung zugeführt worden sei. Der Antragsteller befürchtet, dass mit einer Annahme der Tarifstruktur Alpha 2.2 unter solchen Vorzeichen Tür und Tor geöffnet würden, um via Globalbudget, dessen Einführung nach wie vor drohe, zu einer Rationierung der Leistungen zu kommen, unter welcher in erster Linie die Patienten zu leiden hätten. Er appelliert an das Verantwortungsbewusstsein der Delegierten und ruft sie auf, sich nicht unter Zeitdruck setzen zu lassen.

Der Präsident erwidert, dass die Version «Alpha 2.2" keineswegs noch so offen sei, wie dies der Vorredner zu verstehen gebe. Der grösste Teil ist fixiert, es bleiben nur noch einige hundert Einzelleistungen zu korrigieren. Unter anderem werden noch vor Inkraftsetzung der Tarifstruktur die Nomenklatur für die Gynäkologie überarbeitet und die wirtschaftliche Überprüfung der Radiologie-Institute vorgenommen. Was die Anträge der FMS betrifft, werden diese im Lichte der Daten der Tarifstruktur TarMed geprüft, was bedeutet, dass, wenn man sich einmal über die Grundsätze einig ist, die Kalkulation innert Minuten erfolgen kann. Nun zu den Konsequenzen, welche eine Annahme des Antrages von Dr. Streit zur Folge hätte: die Revisionsrunde 2000 wäre damit vom Tisch; desgleichen das FMS-Modell und wahrscheinlich auch alles andere; man könnte dann nur noch auf die Entscheidung der offiziellen Instanzen warten. Demgegenüber hat sich der Zentralvorstand befleissigt, in seinen eigenen Antrag die zusätzlich eingetroffenen Anträge zu integrieren, wovon sich jedermann beim Durchlesen überzeugen kann. Im übrigen befinden wir uns nicht in einem Stellungskrieg, sondern in einem Bewegungskrieg, d.h. in einem Entwicklungsprozess, im Laufe dessen bereits substantielle Verbesserungen erreicht werden konnten.

In der nachfolgenden Diskussion gibt Dr. $W$. Grete, Präsident der Ärztegesellschaft des Kantons Zürich, zu verstehen, dass man heute nicht zusammengetreten ist, um einen definitiven Tarif zu genehmigen, sondern um die Absicht auszudrücken, mit den Verhandlungen fortfahren zu wollen. Das jedoch könne nur geschehen, wenn wir heute ein ganz klares «Ja" zur Tarifstruktur abgäben. Es sei wichtig, diese Vertrauensbotschaft von seiten der Ärzteschaft abzugeben. Dr. Th. Schweizer, BE, anerkennt seinerseits, dass der GRAT-Tarif eine gute Sache ist, sieht jedoch in der Kostenneutralität ein echtes Virus, das wir uns in das System einpflanzen lassen und welches, via Politiker, im direkten Weg zu einer Rationierung der medizinischen Leistungen führt, die wir ablehnen.

Man schreitet zur Abstimmung über den 3. Absatz im Antrag von Dr. R. Streit («Die heute vorliegenden, weitgehend unausgereiften Konzepte und Vereinbarungen, insbesondere das Konzept der sogenannten Kostenneutralität, werden zurückgewiesen.»). Dieser Antrag wird mit überwältigender Mehrheit abgelehnt. 
3.1 Position Kostenneutralität FMH / Einführungskonzept (inkl. Umsetzung Antrag Ramstein)

Diese Problematik gibt wiederum Anlass zu intensiven Diskussionen. Die Delegierten sind im Besitze eines Papiers, welches den Stand des Dossiers per 30. Januar 2000 wiedergibt. Der Präsident gibt zu beachten, dass es falsch wäre, diese Frage nur einseitig $\mathrm{zu}$ betrachten, bietet doch die Kostenneutralität genauso einen Schutz gegen unten, womit auch die Versicherer gebunden sind, insbesondere im Bereich des KVG. Die ganz klare Ablehnung seitens der FMH, dass die Kostenneutralität hintenherum für die Einführung eines Globalbudgets benützt werde, sei mit aller Entschiedenheit bekräftigt worden. Man kann in dem Moment nicht mehr von Globalbudget sprechen, wo als zentraler Wert die Kosten pro Versicherten festgehalten werden. Hinzu kommt, dass man in Richtung einer "vernünftigen» Beobachtungszeit geht. Die Versicherer gehen mit uns einig, dass die neuen Tarife so eingeführt werden sollen, dass sie weder $\mathrm{zu}$ einer Pro-Kopf-Kostensteigerung noch, im Interesse der Patienten, zu einer Rationierung der Leistungen führen.

Dr. U. Nägeli, Präsident der Ärztegesellschaft des Kantons Glarus, erläutert anschliessend die Gründe seines Antrags (Nr. 2 zu Traktandum 3.1), der verlangt, "dass sich die FMH weiterhin im Rahmen ihrer Möglichkeiten dafür einsetzen soll, dass der Taxpunktwert im KVG-Bereich bei Einführung möglichst einheitlich ist, respektive sich in der von der Ärztekammer vom 8.4.99 beschlossenen Bandbreite von +/-4\% bewegt." Nach Meinung des Antragstellers hat man sich seit Unterbreitung seines Antrages unter politischem Druck immer weiter von diesem im vergangenen April gefassten Beschluss entfernt. Mit dem Beharren auf der Kostenneutralität werde sogar die Idee eines betriebswirtschaftlich gerechneten Tarifs pervertiert. Der Antragsteller, welcher eine Entsolidarisierung innerhalb der Ärzteschaft befürchtet, hält sich für seinen Teil an das Prinzip "gleiche Arbeit gleicher Lohn". Man müsse sich fragen, ob man den Glauben an den im TarMed eingebauten Korrekturmechanismus zur Verhinderung allzu grosser Unterschiede bereits verloren habe. Hingegen bestünden in den angrenzenden Gebieten (MUMA, Analysenliste, usw.) noch grosse Unsicherheiten.

Dr. H. H. Brunner gibt zu beachten, dass nur die Technik der Verhandlungen dazu geführt habe, dass einige Bereiche etwas ausgeklammert wurden. Bei der MUMA seien sich die FMH und das KSK einig geworden, dass man die MUMA als Tarifposition in die Tarifstruktur aufnehmen müsse. Einmal mehr läge aber das Problem nicht bei den Verhandlungspartnern, sondern bei den eidgenössischen Behörden, wo man immer doch darüber diskutiere, ob die Medikamentenabgabe auf Niveau Gesetz oder im Rahmen einer Revision der Verordnung zum KVG geregelt werden solle. Die entsprechenden Beschlüsse seien immer noch ausstehend und alles deute darauf hin, dass sie nicht mehr in diesem Jahr fallen würden. Der Präsident erklärt unmissverständlich zuhanden des Protokolls, dass mit Rücksicht auf die Kostenneutra- lität es für den Zentralvorstand undenkbar sei, eine Inkraftsetzung des neuen Tarifs ins Auge zu fassen, ohne dass die Frage der MUMA geregelt sei.

Zugunsten des Antrags von Dr. Nägeli spricht sich auch seine Kollegin, Frau Dr. C. Hefti, GL, aus und macht auf die bereits heute bestehenden Schwierigkeiten aufmerksam, Schweizer Ärztinnen oder Ärzte zu finden, die bereit seien, gut situierte Arztpraxen in ihrem Kanton zu übernehmen. Der TarMed dürfe nicht dazu führen, die mangelnde Attraktivität von peripheren Regionen noch zu verstärken.

Der Präsident stellt fest, dass der Antrag (Nr. 5 zu Traktandum Nr. 3.1) von Dr. F. Hasler, dem Präsidenten des Bündnerischen Ärztevereins, demjenigen von Dr. Nägeli sehr nahe kommt und schlägt deshalb vor, die beiden Anträge zusammen zu behandeln. $D r$. F. Hasler opponiert diesem Vorschlag nicht, da sowohl sein eigener Antrag wie auch derjenige von Dr. Nägeli und der Antrag des Zentralvorstands in dieselbe Richtung gehen, indem bei allen drei eine Frist für eine Konvergenz gesetzt wird. Ein weiteres Argument für eine Verminderung der Bandbreite des Taxpunktwertes bzw. für einen gesamtschweizerischen Tarif liefert Dr. M. Röthlisberger, GR, welcher auf die Häufigkeit aufmerksam macht, mit welcher die Ärzteschaft eines Kantons Patienten aus anderen Kantonen behandeln muss.

Anschliessend ist es an Dr. Bl. Bourrit, dem Präsidenten der Ärztegesellschaft des Kantons Genf, seinen Antrag (Nr. 3 zu Traktandum 3.1) zu begründen. Er verlangt, dass der TarMed abgelehnt werden müsse, wenn am Kostenneutralitätskonzept festgehalten werde, um daraus ein Globalbudget abzuleiten. Der Antragsteller zeigt zwar ein gewisses Verständnis dafür, wenn die Versicherer verhindern wollen, dass die Inkraftsetzung des Tarifs zu einer Kostenzunahme führt, dass es jedoch die langfristige Neutralität oder anders gesagt, die Aufrechterhaltung während der Dauer eines zwingenden Budgets ist, was Probleme macht. Auch wenn die Genfer bereit seien, den TarMed zu akzeptieren, so seien sie ebenso klar gegen eine Kostenneutralität, sogar während 5 Jahren.

Der Präsident sieht sich veranlasst, an dieser Stelle einige Präzisierungen und Betrachtungen über die Feinheiten einer solch komplexen Verhandlung anzustellen, die im vorliegenden Bericht mit entsprechender Diskretion behandelt werden müssen. Halten wir an dieser Stelle deshalb nur fest, dass das Kostenneutralitätskonzept in seiner Version 5.0, welches die Ärztekammer im Juni 1999 abgelehnt hat, nicht mehr aktuell ist, sondern dass die Diskussionen heute über eine andere Vorlage geführt werden, und nicht innerhalb der TarMed, sondern in direkten bilateralen Verhandlungen mit den Versicherern und auf der Basis der Version 4.0 stattfinden.

Der letzte Antrag (Nr. 4 zu Traktandum Nr. 3.1) zur Kostenneutralität stammt von Dr. E. Steinmann, dem Präsidenten der Ärztegesellschaft des Kantons Luzern, der sein Anliegen mit Folien illustriert. In seinem fünf Punkte enthaltenden Antrag verlangt der Autor, dass "innerhalb eines Zeitraums von 5 Jahren die Taxpunktwerte der TarMed-Tarife soweit zu ver- 
einheitlichen sind, dass die verbleibenden regionalen/ kantonalen Differenzen ausschliesslich auf betriebswirtschaftlich validierten regionalen/kantonalen Unterschieden der Kosten für die Leistungserbringung beruhen" (Punkt 1). Zu diesem Zweck sieht der Autor im zweiten Punkt seines Antrages vor, dass " $a b$ Einführung der Tarifstrukturen die TPW TL für die gesamte Schweiz gleich hoch festzulegen und in der Folge nur insoweit anzupassen sind, als dies ausschliesslich betriebswirtschaftliche Überlegungen erfordern. Änderungen im Rahmen der Kostenneutralität sind ausgeschlossen." Die drei übrigen Punkte des Antrags fordern besondere Regelungen für Ärzte und Ärztinnen, die nur die AL/Ärztliche Leistung verrechnen können, eine Überprüfung, ob 14-16 Kantone mit ähnlicher Kostenstruktur einen Verbund mit gleichen Taxpunktwerten bilden können, und schliesslich, dass die FMH beauftragt wird, die Forderung nach einem einheitlichen Taxpunktwert zur Maxime aller Bereiche der standespolitischen Tätigkeit zu machen. Der Antragsteller wird sowohl von der Sorge geleitet, dass allzu grosse Unterschiede in den Taxpunktwerten zwischen Regionen und Kantonen die ursprüngliche Idee des GRAT-Projektes torpedieren, als auch, dass eine vernünftige Übergangszeit zustande kommt, bis sich die geforderte Konvergenz realisieren lässt.

Der Präsident H. H. Brunner gibt zu beachten, dass es zweifelsohne irrealistisch sei, eine Konvergenz bei Inkraftsetzung realisieren zu wollen, ohne damit nicht ganz massive Spannungen innerhalb der Ärzteschaft und der FMH heraufzubeschwören und damit für zahlreiche Krankenkassen unüberwindliche Probleme zu schaffen. Auch die zumindest kurzfristigen politischen Oppositionen gegenüber einer solchen Konvergenz sind allseits bekannt. Hingegen ist eine Konkretisierung dieser Idee längerfristig durchaus vorstellbar. Man müsste also etappenweise vorgehen und sich entscheiden, ob man sich an die Komponenten der Technischen Leistung hält oder an diejenigen der Ärztlichen Leistung. Dies seien die Fragen, die sich hier konkret stellten.

Die allgemeine Diskussion wird durch Dr. $W$. Grete, $\mathrm{ZH}$, eröffnet, welcher liberalen, weicheren Lösungen den klaren Vorzug vor zentralistischen Lösungen gibt. Unsere Alliierten seien weder in Bern noch in Solothurn zu finden, sondern in jedem einzelnen unserer Kantone, angefangen bei den Kantonen in ihrer Eigenschaft als Verantwortliche für ein gutes Funktionieren ihrer Spitäler. Der Präsident der Ärztegesellschaft des Kantons Waadt, Dr. D. Laufer, unterstützt seinen Vorredner, indem er zu beachten gibt, dass die Ärzte die einzigen seien, die wüssten, wieviel sie verdienten (und verdienen werden), während nur detaillierte Feldstudien die künftige Kostenentwicklung klar aufzeigen könnten. Die Kosten werden nicht nur durch die Ärzte bestimmt, sondern auch durch die Spitäler, die Polikliniken und durch das Verhalten der Patienten. Die Sachen müssen sich einspielen, zumindest vorerst in den Kantonen. Deshalb unterstützt der Redner auch die Initiative von Dr. Steinmann, damit das KSK vernünftige Erklärungen bezüglich der fortbestehenden Kostendifferenzen von einem Kanton zum anderen verlangen dürfe; eine Zeitspanne von 5 Jahren erscheint angemessen, um zu allfälligen Anpassungen zu kommen, aber auch, um eine Lösung für das Problem der Medikamentenabgabe zu finden.

Dr. J. Ammann von der Schweizerischen Gesellschaft für Chirurgie und Präsident der FMS denkt, dass die Diskussion müssig sei, da man nun über ein von ausgewiesenen Juristen erarbeitetes Rechtsgutachten verfüge. Es sind die Kantone allein, die de lege artis die Kompetenz haben, die Vereinbarungen über den Taxpunktwert KVG in ihrem Hoheitsgebiet zu genehmigen. Dr. H. H. Brunner will diese Einschätzung keineswegs in Frage stellen, bemerkt jedoch dazu, dass man das Problem nicht ganz so einfach lösen könne, insbesondere, weil dem Bundesrat die Kompetenz zusteht, im Falle eines Rekursverfahrens in einem Kanton sich zum Grundsatz vernehmen zu lassen. Dr. E. Steinmann, $L U$, appelliert noch einmal an die Solidarität, während $D r$. R. Streit, $B E$, in den hier aufgezeigten Thesen ein Argument mehr sieht, um seine vorher geäusserten Befürchtungen noch $\mathrm{zu}$ nähren. Er befürchtet insbesondere, dass die absolut notwendige "ceteris paribus"-Klausel fallengelassen werde, wenn die kostenneutrale Einführungsphase auf ein Jahr zurückgestuft werden solle, wie dies der Zentralvorstand in seinem Antrag Nr. 1 zu Traktandum Nr. 3.1 unter Ziff. 3 postuliert. Wir dürfen deshalb nichts genehmigen, ohne die Möglichkeit zu haben, Korrekturen vorzunehmen. Für Dr. A. Haefeli, dem Präsidenten des Aargauischen Ärzteverbands, stehen wir vor dem Dilemma Kostenneutralität oder Globalbudget. Aus diesem Dilemma kommen wir nur heraus, wenn die Beobachtungsphase der Kostenneutralität möglichst kurz ist, und die Instrumente, um sie zu messen, vernünftig sind; dies ist der Punkt, über den wir mit den Versicherern verhandeln müssen. Nachdem er noch einige Argumente vorbringt, die ihm für solche Verhandlungen als gut erscheinen, kommt der Redner auf den Antrag von Dr. E. Steinmann zurück, und postuliert für die Konvergenz der Taxpunktwerte. In seiner Funktion als Präsident der Arbeitsgruppe der Kantonalen Ärztegesellschaften ist Dr. Haefeli der Ansicht, dass die Ärztekammer den kantonalen Organisationen keine ausdrücklichen Befehle erteilen solle, die jene weder ausführen könnten noch wollten. Auf dem Papier scheint eine Dauer von 5 Jahren, um die Vereinheitlichung herbeizuführen, vernünftig. Dies würde aber für eine grosse Zahl von Kolleginnen und Kollegen in zahlreichen Kantonen, die bei weitem nicht nur an den Gestaden des Genfersees situiert sind, tiefgreifende Veränderungen in den Strukturen und im Verhalten mit sich bringen, die einer Revolution gleichkämen. Dr. $R$. Nyffeler, Präsident der Ärztegesellschaft des Kantons Freiburg, bemerkt, dass die einheitliche Tarifstruktur, wie sie im Gesetz vorgeschrieben ist, hauptsächlich dazu dient, die Kosten überwachen zu können. Wir müssen uns also im klaren sein, dass, wenn wir selbst unsere Kostenstrukturen nicht analysieren können, wir keine Möglichkeit haben werden, unsere Tax- 
punktwerte auszuhandeln und die Kosten, die wir verursachen, zu rechtfertigen.

Mit dieser letzten Intervention schliesst die allgemeine Diskussion und es werden jetzt die verschiedenen Unteranträge zum Hauptantrag des Zentralvorstands Nr. $1 \mathrm{zu}$ Traktandum Nr. 3.1 erledigt, bevor dann über diesen Hauptantrag abgestimmt werden kann. Der Präsident H. H. Brunner macht die Delegierten darauf aufmerksam, dass der Zentralvorstand sich bemüht hat, in seinen Antrag zahlreiche eingereichte Anträge einzuschliessen. Er verweist insbesondere auf Ziffer 2 im Antrag des Zentralvorstands ("Die Ärztekammer hält fest, dass die Tarifstruktur TarMed - aktuell Alpha 2.2 - in den Tarifen nur wirksam werden kann, wenn zwischen den zuständigen Kostenträgern einerseits, der FMH andererseits eine vertragliche Vereinbarung hinsichtlich der kostenneutralen Einführung der Tarife vorliegt.") und gibt zu beachten, dass die Ärztekammer heute nicht über diese Forderung hinausgehen kann. Der zweite Punkt, über welchen die Ärztekammer sich äussern muss, ist die Frage, ob man eine Konvergenz der Taxpunktwerte gemäss Antrag von Dr. Steinmann will oder nicht. Der Präsident macht die Delegierten darauf aufmerksam, dass mit einer Frist von 5 Jahren man sich wohl mit der Inkraftsetzung der bilateralen Verträge konfrontiert sehen wird, welche mit ihrer bekannten Vereinheitlichungstendenz die heute zu fassenden Beschlüsse zu simpler Makulatur verkommen lassen könnten. Man sollte sich deshalb fragen, ob es nicht sinnvoll wäre, die Möglichkeit einer Konvergenz der Taxpunktwerte zumindest zu überlegen, wie dies ebenfalls in Ziff. 3 des Antrags des Zentralvorstandes vorgesehen ist. Ein wichtiger Punkt, der noch in den Antrag des Zentralvorstands aufzunehmen ist: die Entschädigung für Leistungen im Rahmen der Medikamentenabgabe (MUMA). Unter diesen Voraussetzungen und unter der Bedingung, dass man in den Kantonen, wo dies noch nicht stattgefunden hat, die Analysenliste einschliesst, wie dies Dr. R. Nyffeler, $F R$, fordert, erklärt sich $D r$. U. Nägeli, GL, bereit, seinen Antrag Nr. 2 zu Traktandum Nr. 3.1 mit dem Antrag des Zentralvorstandes zu verschmelzen, da man ja damit die Gewähr habe, dass der Zentralvorstand sich bemühen werde, die Konvergenz innerhalb einer gewissen Zeitspanne herbeizuführen. Dr. $H$. $H$. Brunner versichert, dass die Frage nach entsprechendem Studium auf den Verhandlungstisch mit dem KSK komme. Dr. F. Hasler, GR, schliesst sich seinerseits bezüglich seines Antrags Nr. 5 zu Traktandum Nr. 3.1 den Überlegungen von Dr. U. Nägeli an.

Man schreitet zur Abstimmung über den oben erwähnten Antrag Nr. 3 zu Traktandum Nr. 3.1 von Dr. Bl. Bourrit, GE. Der erwähnte Antrag wird deutlich und mit klarer Mehrheit abgelehnt.

Anschliessend wird der Antrag Nr. 4 zu Traktandum Nr. 3.1 von Dr. E. Steinmann, LU, zur Abstimmung gebracht. Er wird mit 91 gegen 54 Stimmen ebenfalls abgelehnt. Der Präsident kommentiert dieses Resultat dergestalt, dass damit ein Zeichen gesetzt worden sei, dass aber die Frage der Konvergenz auf dem Tisch bleibe, wie man dies vorher gesagt habe.
Schliesslich folgt die Abstimmung über den Antrag Nr. 1 zu Traktandum Nr. 3.1 des Zentralvorstands zur Kostenneutralität, angereichert mit den Elementen aus der Diskussion, was die MUMA und die Analysenliste betrifft. Dieser Antrag wird mit 151 gegen 9 Stimmen angenommen.

\subsection{Umsetzung qualitative Dignität und}

3.3 Überarbeitung 2000

Das Wort hat Dr. M.-G. Schwöbel, Schweizerische Gesellschaft für Kinderchirurgie, zur Begründung seines Antrags Nr. 2 zu Traktandum Nr. 3.2. Er beantragt, dass «die Bezeichnung (Kinderchirurgie» bei allen Einzelleistungen, welche durch Kinderchirurgen erbracht werden, erwähnt sein muss.» Der Antragsteller ist sich bewusst, dass dies als Detailfrage erachtet werden kann, sie hat jedoch für seine Fachgesellschaft eine ganz grundsätzliche Bedeutung. Er erwähnt, dass die qualitative Dignität «Kinderchirurgie» unter 4000 Positionen nur viermal vorkomme. Alle anderen Positionen werden durch eine allgemeine Klausel abgedeckt, was nicht genügend ist. In der Tat werden zahlreiche Eingriffe exklusiv von Kinderchirurgen erbracht und gehen verloren, wenn sie nur noch ausdrücklich in einer Präambel erwähnt sind. Die Kinderchirurgen verlangen deshalb, dass einem Vertreter ihrer Gesellschaft die Möglichkeit eingeräumt wird, im Rahmen der Revision 2000 sämtliche Einzelleistungen $\mathrm{zu}$ überprüfen, und bei denen, welche die Kinderchirurgen betreffen, den Begriff "Kinderchirurgie» hinzuzufügen. Dr. H. H. Brunner, der bereits auf den nächsten, von derselben Fachgesellschaft eingereichten Antrag Nr. 3 zu Traktandum Nr. 3.2 (er verlangt eine Änderung von Ziff. 77 der Generellen Interpretationen) vorgreift, denkt, dass die Fälle, auf welche der Antragsteller in seinen beiden Anträgen hinweist, vor allem in den Technischen Leistungen der Nomenklatur vorkommen. Man habe in der Tat versucht, die Dinge zu vereinfachen, denn neben den Kinderchirurgen hätten nur sehr wenige Fachgesellschaften derart viele Leistungen in allen Kapiteln und es wäre nicht sachdienlich gewesen, hier Limitationen einzufügen. Es wäre hingegen absolut falsch, darin eine Misskreditierung der Tätigkeit der Kinderchirurgen zu sehen. Weder der Zentralvorstand noch die TarMed würden deshalb den beiden Anträgen für die Revisionsrunde 2000 opponieren. Trotz dieser Erklärungen hält Dr. M.-G. Schwöbel daran fest, die Ärztekammer über seine beiden Anträge abstimmen zu lassen, damit in aller Klarheit festgehalten werde, dass die Kinderchirurgen die gleichen Rechte wie alle übrigen Fachgesellschaften der FMH hätten.

Man schreitet deshalb zur Abstimmung über die Anträge Nr. 2 und Nr. 3 zu Traktandum Nr. 3.2. Beide Anträge werden nacheinander praktisch einstimmig angenommen.

Dr. M. Battaglia, Co-Präsident des VSAO, ist der Meinung, dass man wohl die vorliegenden Konzepte zur Kenntnis nehmen könne, dass es aber verfrüht sei, sie jetzt schon zu genehmigen, da man bisher nicht die Möglichkeit gehabt habe, über konkrete Ergeb- 
nisse, insbesondere bezüglich der wohlerworbenen Rechte und des Status der FMH-Titelträger gegenüber den Nicht-Titelträgern zu diskutieren. Die Anwendung des Dignitätskonzeptes sei für die jungen Ärztinnen und Ärzte von ebenso grosser Wichtigkeit, wie es das Neutralitätskonzept für die etablierte Ärzteschaft sei. Der VSAO könne seine Zustimmung zur Tarifstruktur geben, akzeptiere auch, dass man sich in einer "work in progress"-Situation befinde, sehe sich aber ausserstande, einem Konzept zuzustimmen, das noch in seinen Kinderschuhen stecke, was vom Präsidenten bestritten wird, obwohl er zugibt, dass hier noch Massnahmen zu treffen sind, insbesondere im Bereich von Übergangsbestimmungen.

Dr. L. Dubs, Präsident der Schweizerischen Gesellschaft für Orthopädie, stellt fest, dass seine zwei fristgerecht eingereichten Anträge zu Traktandum 3.2 nicht die Gnade gefunden hätten, in den Antrag des Zentralvorstands aufgenommen zu werden; er kündigt an, dass er diese beiden Anträge in der Revisionsrunde 2000 wieder aufnehmen werde, sobald insbesondere Systemfehler in der Anwendung der Dignität ins Spiel kämen.

Nachdem der Präsident präzisiert hat, dass nicht alle Fehler, die zweifelsohne korrigierbar sind, zwingend auf irgendwelche Machenschaften oder Willkür zurückzuführen sind, schreitet man zur Abstimmung über den Antrag des Zentralvorstands Nr. 1 zu Traktandum Nr. 3.2. Der aus drei Punkten bestehende Antrag verlangt im wesentlichen von der Ärztekammer, in zustimmendem Sinne Kenntnis vom Konzept "Umsetzung qualitative Dignität» vom 23.12.1999 zu nehmen, unter Vorbehalt einer Umsetzung der an der Präsidentenkonferenz vom 13.1.2000 überwiesenen Anträge. Weiter wird der Zentralvorstand verpflichtet, die Ärztekammer regelmässig über die Umsetzung des Konzeptes zu informieren und dem Datenschutz höchste Priorität einzuräumen, insbesondere dafür besorgt zu sein, dass keine elektronischen Datenbanken mit Angaben über die Mitglieder in den Besitz Dritter gelangen. Dieser Antrag wird mit überwältigender Mehrheit angenommen.

Der Antrag Nr. 1 zu Traktandum Nr. 3.3 von Dr. $R$. A. Steiner, dem Präsidenten der Schweizerischen Gesellschaft für Gynäkologie und Geburtshilfe, welcher verlangt, dass unter Mitarbeit operativ tätiger Fachgesellschaften eine Sparte «Praxisoperationssaal» zu definieren sei, die nicht praxisfremd ausfallen dürfe, wird grossmehrheitlich angenommen, um so mehr, als der Präsident ihn seitens des Zentralvorstandes als unbestritten bezeichnet hatte.

Die Reihe ist am Antrag Nr. 2 zu Traktandum Nr. 3.3, eingereicht von Prof. T. Rüedi, dem Präsidenten der Schweizerischen Gesellschaft für Chirurgie, und an der Sitzung begründet durch Dr. J. Ammann. Der von der FMS unterstützte Antrag verlangt im wesentlichen, dass mit allen Tarifpartnern vertraglich sicherzustellen sei, dass die Ergebnisse der Überarbeitungsrunde 2000 in die TarMed-Tarifstruktur einfliessen werden. Nach Meinung des Redners könnten mit einer Annahme dieses Antrags die eingangs der Sitzung von Dr. R. Streit geäusserten Befürch- tungen etwas geschmälert werden. Dr. H. H. Brunner nimmt namens des Zentralvorstands diesen Antrag entgegen. Er weist darauf hin, dass die Revisionsrunde 2000 Teil der Tarifstruktur sei, dass dies im Rahmenvertrag mit dem KSK so vorgesehen sei und schliesslich, dass die Agenda der Verhandlungen mit den Vertragspartnern nach der heutigen Sitzung festgelegt werde.

\subsection{Struktur / Vertrag der TarMed-Nachfolge- organisation}

Dr. P. Neidhart von der Schweizerischen Gesellschaft für Anästhesie und Reanimation erläutert den Antrag Nr. 2 zu Traktandum Nr. 3.4, eingereicht von seinem Kollegen Dr. Meister. Mit diesem Antrag wird verlangt, "der TarMed-Vertrag (inkl. Statuten des Vereins (TarMed` sowie des «Organisationsreglementes Verein TarMed») seien zurückzuweisen." Die Antragsteller sind der Meinung, dass sich die Rechtsform "Verein" für eine Organisation wie "TarMed" nicht eignet, weil ein Vereinsmitglied immer die Möglichkeit eines Austritts hat, weil sich die Stimmenverhältnisse zuungunsten der FMH auswirken können und weil man hier frisch-fröhlich Vertragsrecht und Vereinsrecht vermischen will. Der Präsident erklärt ohne Umschweife, dass er diesem Antrag opponieren müsse, da nach monatelangen Verhandlungen diese Rechtsform als die am wenigsten schlechte erachtet werden musste, nachdem man die Rechtsform der Einfachen Gesellschaft definitiv habe aufgeben müssen. Im übrigen müssten die Beschlüsse einstimmig gefasst werden, was die Gefahr einer Minorisierung doch stark relativiere.

Nach dieser Erklärung wird der Antrag Nr. $2 \mathrm{zu}$ Traktandum Nr. 3.4 zur Abstimmung gebracht und mit grosser Mehrheit abgelehnt.

Man kommt anschliessend zum Antrag Nr. $3 \mathrm{zu}$ den Traktanden Nr. 3.4, 3.5, 6 und 7, der von $D r$. G.-P. Jenny, Schweizerische Gesellschaft für Ophthalmologie, erläutert wird. Der Antragsteller will die Statuten der TarMed-Nachfolgeorganisation und den Vertragstext der TarMed-Verträge so abändern, dass a) im Vorstand von jeder betroffenen Fachgesellschaft ein Mitglied neben dem FMH-Verhandlungsmitglied Einsitz nimmt, b) das Mitglied der von Tariffragen betroffenen Fachgesellschaft mit gleichem Stimmrecht und gleichen Kompetenzen ausgestattet wird wie das FMH-Mitglied. Der Antragsteller sieht darin eine Sicherstellung, mit Plausibilitätskontrollen verschiedene Ungereimtheiten im Tarif, die seine Fachgesellschaft im Laufe der Verhandlungen hat feststellen müssen, ausmerzen zu können.

Dr. H. H. Brunner denkt, dass Punkt a) kaum in Frage kommt; er opponiert hingegen Punkt b) betreffend der Delegationen nicht. Die Delegierten schliessen sich seiner Ansicht an und lehnen Punkt a) einstimmig $a b$, während sie Punkt b) mit grosser Mehrheit zustimmen.

An die Reihe kommt jetzt der Antrag des Zentralvorstands, Nr. 1 zu Traktandum Nr. 3.4, dessen Wortlaut hier zur besseren Verständlichkeit des Nachfolgenden wiedergegeben wird: 
"1. Die Ärztekammer nimmt in zustimmendem Sinne Kenntnis vom Stand der Verhandlungen betreffend die TarMed-Nachfolgeorganisation.

2. Der ZV wird aufgefordert, die an der Ärztekammer getroffenen Beschlüsse betreffend die TarMed-Nachfolgeorganisation in den laufenden Verhandlungen umzusetzen. Insbesondere ist dem Prinzip der einstimmigen Beschlussfassung Nachachtung zu verschaffen.

3. Die zur Finanzierung der TarMed-Nachfolgeorganisation notwendigen Mittel müssen im Rahmen der ordentlichen Budgetdebatte durch die Ärztekammer für das nachfolgende Jahr in vollem Umfang und abschliessend beschlossen werden.

4. Unter Voraussetzung einer Erfüllung der Punkte 2 und 3 wird der ZV ermächtigt, die notwendigen Verträge abzuschliessen."

Dr. R. Streit, $B E$, beantragt, Ziff. $4 \mathrm{zu}$ streichen, da es seines Erachtens an der Legislative und nicht an der Exekutive ist, solche Verträge abzuschliessen. Der Präsident H. H. Brunner opponiert diesem Antrag, weil diese Verträge praktisch schon unter Dach und Fach seien und nur noch die Anhänge fehlten; man solle sich hüten, aus übertriebenem Misstrauen heraus, unnötig die Konstituierung der neuen Organisation hinauszuzögern. Demgegenüber und in bezug auf Ziff. 3 präzisiert der Präsident zuhanden von Dr. F. Bossard, dem Präsidenten der Schweizerischen Gesellschaft für Medizinische Radiologie, dass sich das Budget für das erste Jahr in der Grössenordnung von 150000 bis maximal 200000 Franken bewegen werde.

Nach einigen Scharmützeln über die Rechtmässigkeit einer sofortigen Beschlussfassung über dieses Budget kommt der Antrag des Zentralvorstands zur Abstimmung, welche in zwei Schritten durchgeführt wird. Zunächst lehnen die Delegierten den Antrag von Dr. R. Streit (Streichung von Punkt 4) mit grosser Mehrheit ab, anschliessend genehmigen die Delegierten mehrheitlich den Antrag des Zentralvorstandes in seinem vollen Umfang.

\subsection{Struktur / Procedere der Verhandlungs- delegation FMH}

Der in 4 Punkte gegliederte Antrag des Zentralvorstands (Nr. $1 \mathrm{zu}$ Traktandum Nr. 3.5) beantragt der Ärztekammer die Billigung des Konzeptes «Struktur Tarifdienst/Verhandlungsdelegation (1), sieht vor (2), dass "die Delegation für Verhandlungen hinsichtlich Neubewertung operativer und interventioneller Leistungen gemäss FMS-Kostenmodell vom 6.12.99 wie folgt zusammengesetzt wird:

- Leitung durch Delegierten des Zentralvorstands

- 7 Mitglieder, bestimmt durch FMS

- 2 Mitglieder, bestimmt durch KHM

(Kollegium für Hausarztmedizin)

- 1 Mitglied, bestimmt durch FMC

(Foederatio Medicorum Curantium)"

Der Antrag verlangt im weiteren (3), dass «die Überarbeitungsrunde 2000 der Verhandlungsdelegation TarMed-Nachfolgeorganisation übergeben wird, und dass «an allen Verhandlungen bzw. Bereinigungen maximal drei Delegierte der zuständigen/Antrag stellenden Fachgesellschaft zu deren Lasten teilnehmen" sowie (4) dass die 10 Mitglieder des Beirats der Fachgesellschaften anlässlich der ordentlichen Ärztekammer vom Juni 2000 gewählt werden.

Dr. B. Meister, Präsident der Schweizerischen Gesellschaft für Anästhesiologie und Reanimation, sieht für sich die Möglichkeit gekommen, mündlich und illustriert durch Grafiken, die tieferen Gründe aufzuzeigen, die zur Erarbeitung des FMS-Kostenmodells geführt haben. Er zeigt mit aussagekräftigen Beispielen verschiedene Unzulänglichkeiten der TarMedStruktur auf und skizziert, mit welchen Mitteln man hier Abhilfe schaffen und vermeiden könnte, dass die Belegärzte schlicht und einfach von der Bildfläche verschwinden, worunter die Patienten als erste $\mathrm{zu}$ leiden hätten. Der Redner schliesst seine Ausführungen damit, dass die Gefahr einer Spaltung innerhalb der Ärzteschaft für die Annahme dieses Antrages spreche.

Der Präsident bezieht sich auf frühere Erläuterungen, die bereits klar aufzeigten, dass die Entschädigung und die Gewichtung eines Teils der interventionellen und chirurgischen Leistungen alles andere als optimal seien, insbesondere eine Vielzahl von Interventionen mit niedriger Dignität. Dieses Problem sei in fortschreitendem Masse von den Verhandlungspartnern anerkannt worden und es sei jetzt auch die Bereitschaft bei den Versicherern vorhanden, hier Abhilfe zu schaffen. Er unterstreicht, dass das FMSModell eine brauchbare Grundlage für diese Korrekturen darstellt, insbesondere deshalb, weil dafür die Elemente der TarMed-Struktur verwendet wurden, was technisch gesehen von grossem Vorteil ist. Andererseits, da die Grundversorger, falls die Ärztekammer sich für einen Wert von "1.2" für die Variable des Dignitäts-Koeffizienten aussprechen sollte, verlangten, dass diese Lösung befristet und danach durch ein anderes Modell zu ersetzen sei, müsste (sogar zwingend) dieses neue Modell von der neuen, aus den Verhandlungen über das FMS-Modell resultierenden Gewichtung aufgenommen werden. Schliesslich kann festgestellt werden, dass der klare Wille vorhanden ist, ein Ungleichgewicht - klar erkannt und bezeichnet -, welches sich zuungunsten der Gewichtung operationeller und invasiver Leistungen in die Tarifstruktur eingeschlichen hat, $\mathrm{zu}$ kompensieren. Dr. H. H. Brunner unterstreicht an dieser Stelle, dass man die Begriffe "Preis der Leistung" und «Einkommensminderung" nicht miteinander verwechseln dürfe. Sollte der Antrag des Zentralvorstands angenommen werden, würde er integral in den Hauptantrag des Zentralvorstands zur Tarifstruktur Alpha 2.2 aufgenommen; es sei deshalb nicht mehr als normal, dass die FMS in den vorgesehenen Verhandlungen, die nun ohne weitere Verzögerung stattfinden müssten, stark vertreten sein müsse.

Auf eine Bemerkung von Dr. R. Nyffeler, FR, hin, der wünscht, dass man nun schnellstens zu den "Warenkorbstudien» übergehe, statt sich noch länger über hier und da allfällig eintreffende Einkommens- 
einbussen auszulassen, ergreift der Präsident zum letzten Mal unter diesem Kapitel das Wort. Er präzisiert, dass er in seiner Qualität als "Ingenieur" in diesem Projekt seit langer Zeit auf zwei Probleme hingewiesen habe, die wegen fehlender Zustimmung seitens der Kostenträger nicht hätten gelöst werden können, bevor die Zahlen auf den Tisch gekommen seien, nämlich: das Problem, welches der Berechnung der Entschädigung für die Technische Leistung im Spital (eigentliches "ceterum censeo» seit drei Jahren!) zugrunde liegt, und jenes des Ungleichgewichtes in der Tarifstruktur. Aus diesen Gründen habe er für die Position der FMS Verständnis. Danach versichert der Redner, der sich immer noch in der Rolle des «TarifIngenieurs" darstellt, dass die Annahme dieses Antrags keine nachteiligen Folgen für die nicht invasiv tätigen Ärztinnen und Ärzte mit sich bringen werde.

Man kommt zur Abstimmung über den Antrag des Zentralvorstands Nr. $1 \mathrm{zu}$ Traktandum Nr. 3.5, der grossmehrheitlich angenommen wird.

N.B.: Es folgt nun die Behandlung der Anträge zu Traktandum 3.9. An dieser Stelle sei darauf verwiesen, dass der Antrag von Dr. R. Streit, BE, über die Traktanden Nr. 3.5 und 3.6 bereits behandelt worden ist, und dass die Anträge über die Traktanden Nr. 3.5, 3.6 und 3.7, welche von den Dres J. Ammann, Schweizerische Gesellschaft für Chirurgie, L. Dubs, Schweizerische Gesellschaft für Orthopädie, und G.-P. Jenny, Schweizerische Gesellschaft für Ophthalmologie, unterbreitet wurden, zu einem späteren Zeitpunkt im Laufe der Sitzung, in Zusammenhang mit Traktandum Nr. 5 (Genehmigung der Tarifstruktur Alpha 2.2) behandelt werden.

\subsection{Informatik-Umsetzung}

Zwei Anträge liegen zu diesem Traktandum vor, welches ein Thema betrifft (Elektronischer Datentransfer und Datenbanken), das, so unterstreicht Dr. H. H. Brunner, von zentraler Bedeutung für die Zukunft der Ärzteschaft ist.

Der erste Antrag (Nr. 1 zu Traktandum Nr. 3.9) von Dr. M. Müller-Friedli, dem Präsidenten der Schweizerischen Gesellschaft für Allgemeinmedizin, verlangt im wesentlichen, dass (1) «die Modalitäten für die Abrechnung auf elektronischen Datenträgern raschmöglichst mit den Versicherern festzulegen sind", dass (2) «durch diese Abrechnung auf elektronischen Datenträgern in keinem Bereich, in dem heute das System des Tiers garant gilt, das System des Tiers payant eingeführt wird», dass (3) "die FMH beauftragt ist, im Einvernehmen mit den Kostenträgern geeignete, unabhängige Datenpools zu schaffen, die sämtliche Kriterien des Daten- und Persönlichkeitsschutzes der Patienten und der Ärzte erfüllen" und schliesslich (4), dass "bis die Abrechnung auf elektronischen Datenträgern für alle möglich ist, auf sämtliche Limitierungen im TarMed-Tarif zu verzichten ist.» Der Autor dieses Antrages erachtet als von höchster Wichtigkeit, dass die Ärzteschaft selber die Bedingungen formuliert, nach welchen diese Datentranfers stattfinden sollen, um so mehr, als Berater und Informatiker um die Wette Offerten unterbreiten.
Dr. H. H. Brunner erklärt, dass der Zentralvorstand sich in keiner Weise diesem Antrag widersetzt, worauf die Ärztekammer den Antrag stillschweigend genehmigt.

Der zweite Antrag (Nr. 2 zu Traktandum Nr. 3.9) stammt von Dr. P. Meyrat von der Ärzte-Gesellschaft des Kantons Solothurn und verlangt, dass «bei Streitigkeiten zwischen Kostenträgern und Leistungserbringern dem einzelnen Leistungserbringer das Recht auf Gegendarstellung der Tatsachen anhand des eigenen Datenmaterials einzuräumen ist".

Dr. H. H. Brunner sieht sich in der Lage, den Antragsteller dahingehend zu beruhigen, als in dem zwischen FMH und KSK abzuschliessenden Rahmenvertrag insbesondere die Kriterien bezüglich der Überprüfung von Wirtschaftlichkeit, Angemessenheit und Wirksamkeit der Leistungen im gegenseitigen Einverständnis festgelegt werden. Der Antrag von Dr. Meyrat kann demzufolge als stillschweigend angenommen betrachtet werden.

N.B.: An dieser Stelle wird das Traktandum Nr. 4 (Ergebnisse der Warenkorbstudien/Feldstudien) übersprungen, damit man sich jetzt dem herausragenden Punkt dieser Sitzung widmen kann, nämlich der

\section{Genehmigung der Tarifstruktur Version Alpha 2.2}

Es wird noch längere Zeit verstreichen, bis man zur Schlussabstimmung über dieses Traktandum wird schreiten können, da vorher noch eine ganze Reihe diesbezüglich unterbreiteter Anträge behandelt werden müssen. Der erste (Nr. 5 zu Traktandum Nr. 5) stammt von $D r$. R. A. Steiner, dem Präsidenten der Schweizerischen Gesellschaft für Gynäkologie und Geburtshilfe und verlangt, angesichts der noch sehr grossen Unsicherheit bezüglich des Inhaltes der Tarifstruktur, Nichteintreten auf dieses Traktandum. Für den Antragsteller sind die Dinge noch derart unausgereift, dass man zum jetzigen Zeitpunkt nicht ernsthaft "Ja" oder "Nein" sagen kann.

Der Präsident bezieht sich auf seine einleitenden Erläuterungen und lädt die Ärztekammer ein, sich ein Nichteintreten auf die Materie gut zu überlegen. Dies würde von unseren Partnern innerhalb der TarMed als "Nein» verstanden und die entsprechenden Konsequenzen sowohl für den Bereich des UV/MV/IV-Arzttarifs als auch den Bereich des KVG seien unmissverständlich zu Beginn der Sitzung aufgezeigt worden, insbesondere die Tatsache, dass der Bundesrat bei Uneinigkeit unter den Vertragspartnern die Tarifstruktur festlegen müsse. Die Aussicht auf ein nach den Empfehlungen des Preisüberwachers herabgesetztes Referenzeinkommen, verbunden mit einer beträchtlichen Erhöhung der Stunden, würde sich sehr rasch konkretisieren. Es gehe hier nicht darum zu drohen, sondern darum, das wahrscheinlichste Szenarium objektiv darzustellen.

Die Diskussion über diesen vom Zentralvorstand entschlossen bekämpften Antrag wird nicht verlangt, so dass man direkt darüber abstimmen kann. Der 
Antrag von Dr. R. A. Steiner wird mit 143 gegen 19 Stimmen abgelehnt.

Die Ärztekammer befasst sich anschliessend mit dem Antrag (Nr. 6 zu Traktandum Nr. 5) von Dr. M. Schwöbel, Schweizerische Gesellschaft für Kinderchirurgie, der kurz und bündig die Rückweisung der Version TarMed Alpha 2.2 verlangt. Auch dieser Antragsteller ist der Ansicht, dass wir noch vor viel zu vielen Unsicherheiten stehen. Überdies habe er das Vertrauen verloren, nachdem er nun schon seit Jahren, ohne sichtbaren Erfolg, auf die zahllosen fachlichen Fehler hingewiesen habe. In dieser Ausgangslage, wo man nur "Ja" oder "Nein" sagen könne, sehe er sich gezwungen, "Nein» zu stimmen. Der Präsident stellt die Tatsache nicht in Abrede, dass die Kinderchirurgen auf zahlreiche Schwierigkeiten gestossen sind, macht aber gleichzeitig geltend, dass man Wege gesucht habe, hier Abhilfe zu schaffen. Dr. J. Ammann, FMS, unterstützt seine armen Verwandten der Chirurgie, die Kinderchirurgen, und schlägt im Sinne eines Kompromisses vor, dass man sie im vorliegenden Tarif in Klammern setzt, wie man dies im Falle der Gynäkologen und der Radiologen auch tut. Der Präsident sieht sich gezwungen, dieses Ansinnen abzulehnen, da das Paket für die Überarbeitungsrunde 2000 bereits geschnürt ist. Die Kinderchirurgen hätten jedoch die Möglichkeit, einige ihrer Anliegen im Rahmen des FMS-Modells geltend zu machen.

Es folgt eine Diskussion über die Art und Weise, wie die Verhandlungen geführt worden sind und darüber, ob die Basis der Fachgesellschaften genügend oder ungenügend in diesen Prozess eingebunden worden sei, was den Präsidenten veranlasst, hier einige «i-Punkte» zu setzen. Frau Dr. Wehren, BS, ruft ihrerseits die Kammer auf, nun endlich für die Struktur TarMed Alpha 2.2 zu stimmen.

Unser Gast, Dr. M. Ganz, Präsident der Schweizerischen Vereinigung der Belegärzte an Privatkliniken, ergreift an dieser Stelle das Wort. Er bedankt sich beim Zentralvorstand, dass in den tragenden Pfeilern des Berufsorgans das System des Belegarztes Unterstützung gefunden habe und dass die Belegärzte, welche in einem normalen Ausmasse zu Lasten der Sozialversicherung arbeiteten, ihre Existenz mit dem neuen Tarif gesichert sähen. Nach dieser Einleitung erwähnt er die nicht gerade ermutigenden Resultate, die aus einer sorgfältigen Studie seiner Gesellschaft über die Version TarMed Alpha 2.2 hervorgegangen sind. Alles deute darauf hin, dass es den Belegarzt, insbesondere den operativ Tätigen, durch die Auswirkungen des Tarifs, wenn nicht sofort, so doch innerhalb von 5 bis 6 Jahren nach dessen Einführung, nicht mehr gäbe. Demgegenüber werde ein wichtiger Teil von operativen Leistungen in der Grundversorgung nur noch in den subventionierten Spitälern durchgeführt werden können. Die nicht-subventionierten Spitäler würden darauf verzichten müssen, so dass Wartelisten in den öffentlichen Spitälern jetzt schon vorprogrammiert seien. Von der Qualität ganz zu schweigen. Als positiv zu werten, und dafür gebühre dem Zentralvorstand Dank, sei die Tatsache, dass dank der Garantie der wohlerworbenen Rechte den heute im Status des Belegarztes tätigen Ärztinnen und Ärzten Zeit bleibe, sich an die neue Situation anzupassen.

Nach einem weiteren Wortgefecht zwischen verschiedenen Fachgesellschaften und dem Präsidenten, ergreift Dr. M. Schwöbel ein letztes Mal vor der Abstimmung über seinen Antrag das Wort und unterstreicht, dass sein Vorstoss nicht durch pekuniäre Interessen begründet sei, sondern durch die Befürchtung, dass eine Fachgesellschaft schlicht und einfach verschwinde.

In der anschliessenden Abstimmung wird der Antrag Nr. 6 zu Traktandum Nr. 5 mit 135 zu 30 Stimmen abgelehnt.

In der weiteren Verhandlung über Traktandum Nr. 5 stellt der Präsident mit Einverständnis von $D r$. B. Hanimann, dem Präsidenten der Schweizerischen Gesellschaft für Kinderchirurgie, fest, dass dessen Antrag Nr. 7 zu Traktandum Nr. 5 gegenstandslos geworden ist.

Mit Antrag Nr. 8 zu Traktandum Nr. 5 verlangt der Präsident der Schweizerischen Gesellschaft für Gynäkologie und Geburtshilfe, Dr. R. A. Steiner, dass die Kapitel 22 "Gynäkologie/Geburtshilfe" und 23 "gynäkologisch relevante Positionen" nicht Gegenstand der Abstimmung über die Tarifstruktur Alpha 2.2 seien. Der Präsident ruft die Ärztekammer auf, diesen Antrag vor allem aus Systemgründen entschieden abzulehnen. Die anschliessende Abstimmung ergibt eine grossmehrheitliche Ablehnung.

Die Reihe ist nun am Antrag Nr. 10 zu Traktandum Nr. 5, der vom Präsidenten der Schweizerischen Gesellschaft für Allgemeinmedizin, Dr. M. MüllerFriedli, begründet wird. Ein grosser Teil der Forderungen, die unter diesem sieben Punkte umfassenden Antrag gestellt werden, sind bereits anlässlich der Präsidentenkonferenz diskutiert und in der Folge in den Wortlaut des Antrages des Zentralvorstands integriert worden. Der Redner erinnert daran, dass die Grundversorger nur unter Vorbehalt der Neubewertung der interventionellen und operativen Leistungen, wie sie beim Übergang von der Version Beta 3 zur Version Alpha 2.2 vorgenommen wurde, zugestimmt haben. Der Antrag der SGAM kommt also einer Unterstützung der FMS gleich, auch wenn man gleichzeitig fordert, dass innerhalb von zwei Jahren alle Noteingriffe plausibilisiert werden. Ebenfalls innerhalb von zwei Jahren ist das nötige Datenmaterial zu erheben, um endlich für jede Fachgesellschaft die zeitliche Belastung und das Einkommen aus der Sozialversicherung und der Privatversicherung getrennt ausweisen zu können. Der Antragsteller verlangt weiter, dass auch die Grundversorger (SGAM, SGIM, SGP) zu den Expertengesprächen zur Überarbeitung des Kapitels "Gynäkologie und Geburtshilfe» sowie zu den Korrekturen in den Sparten der bildgebenden Techniken beigezogen werden.

Der Präsident verkündet, dass er bei diesem Antrag nur über die Punkte abstimmen lässt, die bestritten sind, während die übrigen Punkte als genehmigt zu betrachten seien. Dieses Vorgehen wird von 
den Delegierten stillschweigend genehmigt. Auf eine Intervention von Frau Dr. A.-C. Froidevaux, GE, hin, welche die Frage stellt, mit welchem Recht die Grundversorger verlangten, an den Verhandlungen über verschiedene medizinische Spezialitäten teilzunehmen, gibt der Präsident zur Antwort, dass es sich hierbei um eine langjährige Praxis bei den Expertengesprächen handle, die sich oft als sehr positiv erwiesen habe, in dem Sinne, dass man meist einen Konsens gefunden habe. $D r$. R. A. Steiner, Präsident der Schweizerischen Gesellschaft für Gynäkologie und Geburtshilfe, und Prof. M. Litschgi, der sich ihm später anschliesst, weisen auf dieselbe Problematik bezüglich Punkt 2 des Antrags der Grundversorger, Nr. $10 \mathrm{zu}$ Traktandum Nr. 5, hin und befürchten ein externes "Diktat" auf die sie besonders betreffenden Leistungen, dies um so mehr, als sie selbst nie von der Gegenseitigkeit bei den Verhandlungen ihrer «Nachbar»-Gesellschaften hätten profitieren können. Dr. Bl. Bourrit, GE, drückt aus, dass er als Gynäkologe im vorliegenden Falle nicht anders könne, als das Ganze in einem breiteren Kontext zu sehen, man denke nur zurück an das Los, das im vergangenen Jahr dem Weiterbildungsprogramm der Gynäkologen in der Ärztekammer beschieden worden sei. Es folgen noch weitere Wortgefechte, bevor Dr. J. de Haller, SGAM, dem das letzte Wort in dieser Kontroverse zusteht, erklärt, dass der Antrag seiner Fachgesellschaft eine Einheit darstelle, deren Zerschlagung die SGAM nicht akzeptiere.

Es folgen die Abstimmungen. In einer ersten $A b$ stimmung lehnt die Ärztekammer mit 100 gegen 45 Stimmen die Streichung von Ziff. 2 des SGAM-Antrages $a b$. In der Schlussabstimmung wird der integrale Antrag der SGAM mit grosser Mehrheit angenommen.

Es folgt Antrag Nr. 11 zu Traktandum Nr. 5 von Prof. T. Rüedi, Schweizerische Gesellschaft für Chirurgie. Dieser Antrag, der in der Sitzung von $D r$. J. Ammann, FMS, begründet wird, verlangt, dass «die Sozialversicherungstarife (UV/MV/IV und KVG) das FMS-Tarifmodell für die Berechnung invasiver und operativer Leistungen spätestens bei deren Inkrafttreten zwingend einschliessen.» Wie der Präsident bemerkt, indem er sich auf die früheren Diskussionen betreffend der FMS-Modells bezieht, ist sich der Zentralvorstand bewusst, dass die diesbezüglichen Verhandlungen unverzüglich beginnen müssen. Die Ärztekammer genehmigt stillschweigend den Antrag von Prof. T. Rüedi, wie dies vom Präsidenten beantragt wird.

Mit Antrag Nr. 9 zu Traktandum Nr. 5 verlangt Dr. F. Bossard, Schweizerische Gesellschaft für Medizinische Radiologie, dass die Kapitel «Radiologie und bildgebende Techniken" inkl. "Nuklearmedizin" aus dem heutigen Ärztekammerentscheid über die Tarifstruktur Alpha 2.2 ausgeklammert werden. Der Antragsteller erklärt, dass es den Radiologen nicht nur um die Frage der finanziellen Bewertung der Technischen Leistungen gehe, sondern vielmehr um grundsätzliche Fragen, neben Detailfragen. Es seien also noch viele Punkte offen, die knapp angedeutet, aber nicht namentlich im Antrag des Zentralvorstands Nr. $1 \mathrm{zu}$ Traktandum Nr. 5 aufgeführt seien, wie dies seine Fachgesellschaft an der Präsidentenkonferenz vom 13. Januar 2000 verlangt habe. Der Präsident H. H. Brunner ist bereit, den gesamten Inhalt der Wunschliste der Schweizerischen Gesellschaft für Medizinische Radiologie in das Paket für die Überarbeitungsrunde 2000 aufzunehmen, indem er nebenbei daran erinnert, dass er der erste gewesen sei, der den Finger auf die von den Radiologen aufgezeigten Fehler und Unzulänglichkeiten gelegt und schliesslich die Versicherer dazu gebracht habe, dies zu akzeptieren. Nach dieser Präzisierung sieht er sich gezwungen, aus formalen Gründen, diesem Antrag zu opponieren. Dr. F. Bossard, bestärkt durch die erhaltenen Zusicherungen, zieht seinen Antrag daraufhin zurück.

Mit Antrag (Nr. $12 \mathrm{zu}$ Traktandum Nr. 5) von Dr. L. Dubs, Schweizerische Gesellschaft für Orthopädie, wird beantragt, dass «die MV und die IV aufzufordern seien, sich zur Tarifstruktur Alpha $2.2 \mathrm{zu}$ äussern, bevor der UV/MV/IV-Tarif in Kraft gesetzt wird.» In der Sprache des Parlamentes würde es sich hier eher um eine Interpellation als um einen Antrag handeln, erklärt Dr. H. H. Brunner und verweist darauf, dass die Zentrale für Medizinaltarife (ZMT) ihrerseits die Tarifstruktur in einer Sitzung im Dezember 1999 genehmigt hat, dass noch eine gewisse Unklarheit bezüglich der abschliessenden Stellungnahme von Militärversicherung (MV) und Invalidenversicherung (IV) vorhanden sei, die sich bekanntlich im Einflussbereich des Eidg. Departementes des Innern befänden. Letzteres müsse also noch Stellung nehmen. Wenn es hier zu Verzögerungen komme, seien diese bestimmt nicht der FMH anzulasten ... Aufgrund dieser Informationen besteht kein Anlass, über den vorliegenden Antrag abzustimmen.

Die Ärztekammer befasst sich anschliessend mit dem Antrag Nr. 13 zu Traktandum Nr. 5, unterbreitet von Prof. T. Rüedi und Dr. J. Ammann von der Schweizerischen Gesellschaft für Chirurgie, der obwohl kurz und bündig gehalten, doch eine Grundsatzfrage betrifft: "Die Tarifstruktur sei dem Bundesrat nicht zur Genehmigung zu unterbreiten." Namens der Antragsteller verweist Fürspr. F. Bernath, Generalsekretär der FMS, auf die wiederholten und konstanten Druckversuche der Vorsteherin des EDI, im Hinblick auf eine Genehmigung der Tarifstruktur durch die Behörden, zuhanden des Bundesrates Auskünfte und Dokumente aller Art zu erhalten. Der Redner hat diese Frage im Detail studiert und ist zum Schluss gekommen, dass diese angebliche Kompetenz des Bundesrates bezüglich der Genehmigung der Tarifstruktur nicht existiert. Die Kompetenz des Bundesrates ist dann gegeben, wenn ein eidgenössischer Tarif existiert - was etwas anderes ist als eine Tarifstruktur -, oder im Falle eines Rekurses gegen einen zustimmenden Entscheid einer Kantonsregierung zu einem Tarif im Bereiche des KVG. Um seine Meinung abzustützen, hat der Redner seine Untersuchung im Sinne einer "Zweitmeinung" drei namhaften Juristen aus Universitätskreisen unterbreitet, die allesamt 
diese Schlussfolgerungen als begründet bestätigt haben. Unter diesem Lichte betrachtet, sind die fortwährenden Druckversuche seitens des EDI gegenstandslos. Der Präsident H. H. Brunner wünscht eine Modifikation dieses Antrages. Er erklärt, dass wir seit langem dieselben Vorbehalte wie Fürspr. Bernath haben (s. Publikation von Fürspr. Kuhn, Schweiz. Ärztezeitung 1999;80[28]:1728-35), und dass wir immer wieder, mündlich und schriftlich, vom EDI Art und Grundlage dieses Genehmigungsverfahrens verlangt haben. Wir haben bis heute keine Antwort erhalten, was nicht unbedingt ein Zufall sein muss ... Der Redner verweist darauf, dass er auch immer wieder innerhalb der TarMed an dieser Position festgehalten habe, ohne dass man sich ihm angeschlossen hätte.

Im Anschluss an die Diskussion genehmigt die Ärztekammer den Antrag der Schweizerischen Gesellschaft für Chirurgie mit folgendem Wortlaut: «Der Zentralvorstand wird beauftragt, vom EDI im Sinne eines Ultimatums die notwendigen Stellungnahmen zu verlangen und die Tarifstruktur dem EDI und dem Bundesrat so lange nicht zu unterbreiten, als diese Situation nicht abschliessend geklärt ist." Dieser Antrag, der in dieser Form von Fürspr. Bernath namens seiner Auftraggeber akzeptiert wird, darf die Ärztekammer nicht daran hindern, am heutigen Tag definitiv über die Tarifstruktur Alpha 2.2 zu beschliessen, erklärt Dr. H. H. Brunner im Sinne einer Schlussfolgerung zu diesem Traktandum.

Dr. Chr. Ramstein, Präsident der Ärztegesellschaft des Kantons Solothurn, unterbreitet einen aus zwei Punkten bestehenden Antrag (Nr. 14 zu Traktandum Nr. 5), dessen erster Punkt genau dem Wortlaut des Antrages des Zentralvorstands Nr. 1 zu Traktandum Nr. 5 entspricht, während Punkt 2 daran erinnert, dass "Voraussetzung für die gesamtschweizerische Umsetzung und Anwendung dieser Tarifstruktur jedoch der rechtsgültige Abschluss eines Rahmenvertrages (Tarifvertrag) zwischen $M T K / I V / B A M V$ und FMH einerseits sowie zwischen KSK und FMH andererseits, aber auch der Abschluss von Tarifverträgen zwischen den Kantonalverbänden der Krankenversicherer und den kantonalen Ärztegesellschaften ist." Der Präsident H. H. Brunner macht geltend, dass das Wesentliche dieses Antrages in demjenigen (Nr. $1 \mathrm{zu}$ Traktandum Nr. 5) des Zentralvorstandes enthalten ist und man deshalb den Antrag von Dr. Ramstein als integriert betrachten kann; der Antragsteller erklärt sich mit dieser Sicht einverstanden, so dass sich eine Abstimmung über seinen Antrag erübrigt.

Man kommt nun zurück auf die wichtigen Grundsatzfragen, die von $D r$. R. Streit, BE, im ersten und zweiten Absatz seines Antrages (Nr. 1 zu Traktanden Nr. 3, 5 und 6) aufgeworfen werden (Absatz 3 wurde bereits zu Beginn der Sitzung abgelehnt). Kurz gesagt verlangt Absatz 1, dass "die Tarifstruktur Alpha zwecks versuchsweiser Einführung während einer einjährigen Pilotphase im UV/MV/IV-Bereich" mit einem Taxpunktwert von Fr. 1.- "genehmigt wird", während Absatz 2 postuliert, dass man erst verbindlich über die definitive Übernahme der Tarifstruktur, insbesondere für den KVG-Bereich, entscheidet, wenn die Ergebnisse der Pilotphase im UV/MV/IV-Bereich vorliegen. Der Antragsteller ruft die Kammer mit Entschiedenheit dazu auf, seine Ansicht zu unterstützen, wenn sie nicht wolle, dass die Ärzteschaft «die Katze im Sack kaufe" und sich für die Zukunft definitiv binde. Er stellt in Abrede, dass dieses Vorgehen einem "Nein" gleichkomme, was seiner Meinung nach nur eine subjektive Interpretation wäre. Dieser Meinung ist Dr. H. H. Brunner nicht, der im übrigen daran erinnert, dass wir uns die Möglichkeit eingeräumt hätten, den Vertrag kurzfristig zu kündigen. Dr. J. Ammann, FMS, unterstützt den Antrag von Dr. Streit, denn, obwohl er nicht «Nein» stimmen werde, möchte er doch noch vor einer definitiven Annahme einige Zusicherungen erhalten. Dies sei im übrigen der Grund, weshalb die FMS eine nicht unmittelbar auf die Beschlüsse der Ärztekammer folgende Urabstimmung wolle, sondern einer Urabstimmung nach einer gewissen Beobachtungszeit nach Inkraftsetzen dieser Struktur im "Blauen Tarif» den Vorzug gebe, nachdem ja die Präsidentenkonferenz (konsultativ) die Durchführung erst nach einem Jahr abgelehnt habe.

Diese letzte Intervention veranlasst den Präsidenten, die Anträge des Zentralvorstands Nr. 1 zu Traktandum 1 und Nr. 1 zu Traktandum 5 zu erläutern. Die Anträge des Zentralvorstands tragen den wesentlichen, in verschiedensten Strömungen innerhalb der FMH vorhandenen Anliegen (FMS-Modell, Revision 2000) sowie den Anträgen der Ärztekammer bezüglich Genehmigung der Struktur Rechnung. So werde man feststellen, dass in Ziff. 4 des Antrages Nr. 1 des Zentralvorstands zu Traktandum Nr. 5 der Antrag von Dr. Chr. Ramstein, So, aufgenommen wurde, und zwar noch verstärkt, indem man ihn in einen unanfechtbaren Zusammenhang gestellt habe. Zur Urabstimmung: Man wäre sowohl schlecht beraten, sie jetzt sofort durchführen, wie auch, sie erst in einem Jahr stattfinden zu lassen. Wenn das EDI tatsächlich eine Entscheidung fällen will (oder muss), müsste dies im März oder April geschehen, wenn man den Krankenkassen die Möglichkeit geben wolle, mit der Kalkulation ihrer Prämien zu beginnen. Falls die Bedingungen, über welche die Ärztekammer jetzt abgestimmt hat, nicht erfüllt werden, müsste die Urabstimmung unverzüglich gestoppt werden. Dieses Vorgehen ist kommunizierbar und gewährt uns den breitesten Manövrierraum. Der Präsident schliesst, indem er die Kammer aufruft, aus formalen Gründen den Antrag von Dr. R. Streit abzulehnen. Da das Wort nicht mehr verlangt wird, schreitet man zur Abstimmung.

In einer ersten Abstimmung kommt der Antrag von Dr. R. Streit zur Abstimmung. Die Ärztekammer lehnt ihn mit 129 gegen 33 Stimmen, bei einigen Enthaltungen, $a b$.

In einer zweiten Abstimmung ist die Ärztekammer zunächst aufgerufen, über die von drei Delegierten verlangte geheime Abstimmung zu beschliessen. Die geheime Abstimmung kann nicht die geforderte Einviertelmehrheit auf sich vereinen, denn sie wird mit 129 gegen 33 Stimmen, bei 6 Enthaltungen, abgelehnt. 
In einer dritten Abstimmung wird der Antrag des Zentralvorstands Nr. $1 \mathrm{zu}$ Traktandum Nr. 5 mit 144 gegen 30 Stimmen, bei 3 Enthaltungen, angenommen.

Im Hinblick auf eine Urabstimmung wird der Wortlaut hier vollumfänglich wiedergegeben:

Wortlaut, wie er von der Ärztekammer am 2. Februar 2000 genehmigt wurde:

1. Die Ärztekammer vom 2.2.2000 genehmigt die Tarifstruktur TarMed in der Version Alpha 2.2 berechnet zum Ansatz Fr. 1.- pro Taxpunkt, dies ausschliesslich hinsichtlich ihrer vertraglichen Einführung in die kantonalen Tarife gemäss KVG bzw. den UV/MV/IV-Arzttarif und Spitalleistungskatalog durch die zuständigen Kostenträger und Leistungserbringerorganisationen.

2. Der zugrunde gelegte generelle Severity-Complexity Score von 1.2 für operative/interventionelle Leistungen ist auf maximal 2 Jahre begrenzt. Er ist durch einen neuen Berechnungsmodus abzulösen, dessen Basis das FMS-Kostenmodell vom 6.12.99 ist. Die entsprechenden Verhandlungen sind mit den Kostenträgern unverzüglich aufzunehmen und vor Inkraftsetzung der auf der TarMed-Tarifstruktur aufbauenden Tarifverträge abzuschliessen.

3. Die Überarbeitungsrunde 2000 ist integraler Bestandteil der Genehmigung Version Alpha 2.2; die Zustimmung erfolgt vorbehältlich der einvernehmlichen Bereinigung durch Kostenträger und Leistungserbringer für die zu bearbeitenden, in der Datenbank Überarbeitungsrunde 2000 festgehaltenen Leistungen bzw. Kapitel (insbesondere Gynäkologie/Geburtshilfe und bildgebende Techniken/Radiologie).

4. Die vertragliche Einführung gemäss Ziffer 1 beinhaltet als conditio sine qua non eine vertragliche Vereinbarung zwischen den zuständigen Leistungserbringern und Kostenträgern betreffend die sog. kostenneutrale Einführung der Tarife. Diese Vereinbarung muss verbindliche Vorgaben hinsichtlich einer gesamtschweizerischen Konvergenz der Taxpunktwerte ärztliche und technische Leistung im Bereich "Arztpraxis» und "Spital ambulant» über einen Zeitraum von 5 Jahren mit einer Varianz gemäss betriebswirtschaftlichen Daten, maximal aber von $+/-4 \%$ um einen mengengewichteten Mittelwert, enthalten.

5. Der Zentralvorstand stellt bis zur nächsten Ärztekammersitzung, spätestens aber bis zur Ärztekammersitzung Juni 2000 Bericht und Antrag zu den Punkten 2-4.

Man kommt nun zur Frage der Urabstimmung. Dazu liefert der Generalsekretär einige Erläuterungen zur praktischen Durchführung. Dr. L. Dubs, Schweizerische Gesellschaft für Orthopädie, zieht seinen Antrag (Nr. 1 zu Traktandum Nr. 5.2) zurück, da dies seiner Meinung nach das ganze Verfahren zu sehr verzögern würde. Es werden noch einige Betrachtungen angestellt und Meinungen geäussert, insbesondere von einigen Delegierten, die eine gewisse Zeitspanne zur
Verfügung haben möchten, um die Basis zu informieren oder bis zum Moment, wo das Ergebnis dieser Konsultation verpflichtend wird. Die Frage wird auch nach den Dokumentationen gestellt, über welche unsere Mitglieder werden verfügen können. Der Präsident präzisiert zuhanden der einen, dass die Urabstimmung dazu dienen werde, den Beschluss, der gerade eben von der Ärztekammer gefasst worden sei und als ein geschnürtes Paket anzunehmen oder abzulehnen sei, zu bestätigen oder aufzuheben, an die Adresse der anderen, dass die spezifischen Schulungskurse über die Struktur Alpha 2.2 bereits begonnen hätten und fortgesetzt würden und, nicht zu vernachlässigen, dass eine umfangreiche Information auf dem «Internet» (www.fmh.ch) vorhanden sei und auch auf einer CD-Rom zur Verfügung gestellt werde. Die Alternative "Gutenberg" wiegt 7,5 Kilo und ist $62 \mathrm{~cm}$ hoch... Dies ist die Konsequenz eines extrem differenzierten Tarifs, wie er, man vergesse das nicht, von den verschiedenen Fachgesellschaften gefordert worden sei. Nun, wir würden mit dem Einverständnis der Fachgesellschaften versuchen, einen Auszug aus den wichtigsten und am häufigsten gebrauchten Leistungen zusammenzustellen und diesen, allenfalls, in der Ârztezeitung zu publizieren. Um noch einmal die zum wiederholten Male geäusserten Befürchtungen von Dr. J. Ammann, FMS, zu beschwichtigen, gibt der Präsident zu verstehen, dass die FMH via Ärztekammer sich jederzeit die Möglichkeit offenhalte, sich ihre Freiheit zurückzuholen, zum Beispiel, wenn etwa die legitimen Forderungen der Gynäkologen und der Radiologen zu wenig berücksichtigt würden. Man verzeichnet noch eine Intervention von $D r$. $R$. Urscheler, dem Präsidenten der Ärztegesellschaft des Kantons St. Gallen, welcher der Ansicht ist, dass die Kammer nicht von sich aus die Durchführung einer Urabstimmung beschliessen sollte, sondern die Verantwortung dafür den gemäss Statuten dazu berechtigten Gesellschaften überlassen solle.

Man schreitet zur Abstimmung, die eine Mehrheit von $91 \mathrm{Ja}$-Stimmen gegen 54 Nein-Stimmen, bei 10 Enthaltungen, ergibt. Wichtiger Hinweis: Wegen einer ärgerlichen Unaufmerksamkeit hatte der Generalsekretär zunächst verkündet, die Ärztekammer habe sich zugunsten einer Urabstimmung ausgesprochen. Dabei wurde nicht beachtet, dass es für einen solchen Beschluss eine Zweidrittelmehrheit braucht. Einige Minuten nach Anzeigen dieses Fehlers wurde das den Statuten entsprechende Ergebnis den Delegierten bekanntgegeben: die Mehrheit von zwei Dritteln war nicht erreicht worden.

\section{Verträge}

Es liegen zwei Anträge mit praktisch identischem Inhalt vor (Antrag Nr. 1 und 2 zu Traktanden 3.4, 3.5, 6, 7), der Erste von Dr. G.-P. Jenny, Schweizerische Gesellschaft für Ophthalmologie, der Zweite von Dr. L. Dubs, Schweizerische Gesellschaft für Orthopädie. Beide sehen im wesentlichen vor, dass die Fachgesellschaften in allen Verträgen die Möglichkeit haben, 
für den Fachbereich, der sie betrifft, den entsprechenden TarMed-Vertrag aufzukündigen. Der Präsident H. H. Brunner gibt zu beachten, dass, und sei dies auch nur aus formellen Gründen, eine Fachgesellschaft nicht aus einem Tarifvertrag aussteigen könne. Dieses Recht steht auf eidgenössischer Ebene der FMH ("Blauer Tarif») für auf kantonaler Ebene ausgehandelte Tarifverträge den Kantonalen Ärztegesellschaften oder einzelnen Ärztinnen und Ärzten zu. Schlussfolgerung: Aufgrund dieser objektiven Erläuterungen insistieren die beiden Antragsteller nicht auf ihrer Forderung.

Auf dem Tisch des Präsidenten liegen nun noch zwei Anträge (Nr. 1 und 2 zu Traktandum Nr. 6.1), unterbreitet von Dr. W. Grete und M. F. Rübel, Präsident bzw. Sekretär der Ärztegesellschaft des Kantons Zürich. Beide betreffen den Wortlaut des erst im Entwurf vorliegenden Vertrags zwischen der FMH und den Versicherern des Blauen Tarifs. Es wäre verfrüht, sie zum jetzigen Zeitpunkt zu behandeln, Dr. H. H. Brunner wird sie jedoch in den kommenden Verhandlungen berücksichtigen und dankt im übrigen an dieser Stelle allen, die ihm Vorschläge haben zukommen lassen.

Was den Rahmenvertrag mit dem KSK betrifft, erwarten die Verantwortlichen für die Verhandlungen von den Kantonalen Ärztegesellschaften klare Hinweise, über das, was in diesem Vertrag die gesamtschweizerische Ebene betreffen und das, was auf der kantonalen Ebene belassen werden soll.

Der abtretende Präsident der Société médicale de la Suisse romande, Dr. A.E. Marmy, verabschiedet sich von der Ärztekammer und ergreift die Gelegenheit, um in Namen aller dem Präsidenten H. H. Brunner für seine enorme Arbeit, die er geleistet hat, zu danken (anhaltender Applaus). Dr. H. H. Brunner freut sich über dieses Kompliment und erklärt, er sei gewillt, solange er noch im Schwung sei, seine Arbeit im Dienste der ganzen Ärzteschaft weiterzuführen und bedankt sich bei allen Teilnehmerinnen und Teilnehmern an dieser Sitzung für ihre konstruktive und effiziente Mitarbeit. Er schliesst in diesen Dank die Mitarbeiterinnen und Mitarbeiter des Generalsekretariats mit ein, die seit Monaten mit ausserordentlichem Einsatz dafür gearbeitet haben, dass diese Sitzung hat stattfinden können (Applaus).

Die Sitzung wird damit, es ist einige Minuten nach 17.00 Uhr, geschlossen. 